\title{
Epicardial infarct repair with basic fibroblast growth factor-enhanced CorMatrix-ECM biomaterial attenuates postischemic cardiac remodeling
}

\author{
Holly E. M. Mewhort, MD, Jeannine D. Turnbull, BSc, H. Christopher Meijndert, MBT, \\ Janet M. C. Ngu, MD, MSc, and Paul W. M. Fedak, MD, PhD, FRCSC
}

\begin{abstract}
Objectives: Dysregulation of extracellular matrix (ECM) following myocardial infarction is a key contributor to myocardial fibrosis, chamber dilation, and progression to heart failure. Basic fibroblast growth factor is a potent inhibitor of fibrosis. We propose a novel surgical procedure leveraging a commercially available ECM biomaterial for the treatment of ischemic heart failure.
\end{abstract}

\begin{abstract}
Methods: Epicardial infarct repair using CorMatrix-ECM biomaterial patch (CorMatrix Cardiovascular Inc, Roswell, Ga) was compared with sham in a rat myocardial infarction model. Key indices of ischemic remodeling, including inflammation, fibrosis, and myocardial performance were evaluated 16 weeks post-treatment.

Results: Histology and immunohistochemistry demonstrated comprehensive integration of CorMatrix-ECM biomaterial patch without evidence of immune reaction and an increase in basic fibroblast growth factor expression in treated animals. Functional analysis by serial echocardiography of normal $(\mathrm{n}=13)$, sham $(\mathrm{n}=15)$, nonenhanced CorMatrix-ECM patch $(\mathrm{n}=18)$, and basic fibroblast growth factor-enhanced CorMatrix-ECM patch $(\mathrm{n}=10)$ animals revealed an improvement in ejection fraction in basic fibroblast growth factor-enhanced CorMatrix-ECM patch animals compared with shams $(55.3 \% \pm 8.0 \%$ vs $35.1 \% \pm 7.6 \%$; $P<.001)$. Prevention of left ventricle remodeling was also confirmed by pressure volume loop analysis, which demonstrated reduced left ventricular end diastolic volumes in basic fibroblast growth factor-enhanced CorMatrix-ECM patch animals $(\mathrm{n}=5)$ compared with shams $(\mathrm{n}=6)(208.0 \pm 59.3 \mu \mathrm{L}$ vs $363.1 \pm 108.7$ $\mu \mathrm{L} ; P<.01)$ and improved left ventricle contractility in nonenhanced CorMatrix-ECM patch $(\mathrm{n}=7)$ and basic fibroblast growth factor-enhanced CorMatrix-ECM patch animals compared with shams $(0.709 \pm 0.306$ and $0.609 \pm 0.160$ vs $0.437 \pm 0.218 ; P<.05)$.
\end{abstract}

Conclusions: Epicardial infarct repair with basic growth factor-enhanced CorMatrix-ECM biomaterial patch attenuates myocardial remodeling and improves cardiac performance after subacute myocardial infarction in a rat coronary ligation model. These observations establish proof-of-concept for this novel surgical approach. (J Thorac Cardiovasc Surg 2014;147:1650-9)

Survival rates following acute myocardial infarction (MI) are improving and the incidence of ischemic heart failure is reaching epidemic proportions. ${ }^{1}$ Interstitial fibrosis and progressive ventricular dilation, 2 hallmarks of ischemic heart failure, are the result of structural remodeling in both the infarcted and remote myocardium. Restoring myocardial perfusion by revascularization after $\mathrm{MI}$ is beneficial but does not prevent maladaptive post-MI remodeling. Adjunctive therapies at the time of surgical revascularization that target the injured region may prevent or limit

From the Campbell Cardiovascular Translational Research Program, Division of Cardiac Surgery, Department of Cardiac Sciences, Libin Cardiovascular Institute of Alberta, University of Calgary, Calgary, Alberta, Canada.

Support for this work was provided by the Heart and Stroke Foundation of Canada. Disclosures: Authors have nothing to disclose with regard to commercial support.

Received for publication June 10, 2013; revisions received July 23, 2013; accepted for publication Aug 2, 2013; available ahead of print Sept 30, 2013.

Address for reprints: Paul W. M. Fedak, MD, PhD, FRCSC, C880, 1403 29th St, NW,

Calgary, Alberta, T2N 2T9, Canada (E-mail: paul.fedak@gmail.com).

$0022-5223 / \$ 36.00$

Copyright (c) 2014 by The American Association for Thoracic Surgery

http://dx.doi.org/10.1016/j.jtcvs.2013.08.005 structural cardiac remodeling and in so doing improve outcomes for patients with ischemic injury.

In our study, we examined the utility of basic fibroblast growth factor (bFGF)-enhanced CorMatrix-ECM biomaterial patches (CorMatrix Cardiovascular Inc, Roswell, Ga) as a novel surgical epicardial patch repair after subacute MI in rodents. To establish proof-of-concept, we determined its biocompatibility in host myocardium, expression of matricellular proteins after patch implantation, and procedural effectiveness in limiting progressive adverse structural remodeling after ischemic injury.

\section{METHODS \\ Experiment Animals \\ The experiment protocol was approved by the Institutional Animal Care Committee. All procedures performed were in accordance with the Guide for the Care and Use of Experimental Animals from the Canadian Council on Animal Care ${ }^{2}$ and the Guide for the Care and Use of Laboratory Ani- mals. ${ }^{3}$ Male rats (Fischer CDF strain) weighing between 170 and $200 \mathrm{~g}$ were obtained from Charles River Canada Inc (Montreal, Quebec, Canada).}




\section{Abbreviations and Acronyms \\ $\mathrm{bFGF}=$ basic fibroblast growth factor \\ $\mathrm{ECM}=$ extracellular matrix \\ MI = myocardial infarction \\ PBS = phosphate buffered saline}

\section{Rat MI Model}

Animals were anesthetized with isoflurane (4\%), intubated, and maintained on continuous ventilation with medical-grade oxygen supplemented with isoflurane ( $1 \%-2 \%)$ for the duration of the surgical procedure. The heart was exposed via a left anterolateral thoracotomy. The pericardium was incised and the left anterior descending artery was identified and proximally ligated using interrupted 7-0 polypropylene sutures. The incision was reapproximated and animals were recovered. Analgesia included buprenorphine (Hospira Healthcare Corp, Lake Forest, Ill) $0.03 \mathrm{mg} / \mathrm{kg}$ preoperatively, 4 to 8 hours postoperatively, and repeated as necessary every 8 hours for the first 24 hours postoperatively; $2 \%$ lidocaine on the endotracheal tube tip at the time of intubation; and $2 \%$ lidocaine within the skin incision before closure. Animals were administered 150,000 IU/kg Duplocillin LA antibiotic (Intervet Canada Ltd, Kirkland, Quebec, Canada) and $10 \mathrm{cc} / \mathrm{kg}$ normal saline $(0.9 \%$ sodium chloride) preoperatively.

\section{CorMatrix-ECM Biomaterial}

A commercially available extracellular matrix (ECM) biomaterial procured from porcine small intestine submucosa was obtained. Nonenhanced CorMatrix-ECM patch was prepared for surgical implantation by soaking it in phosphate buffered saline (PBS) (Lonza Group, Basel, Switzerland) for a minimum of 2 hours at $37^{\circ} \mathrm{C}$. bFGF enhancement was achieved by soaking the CorMatrix-ECM material in $40 \mu \mathrm{L}$ of $25 \mu \mathrm{g} / \mathrm{mL}$ bFGF (Invitrogen Corporation, Carlsbad, Calif) in PBS for a minimum of 2 hours at $37^{\circ} \mathrm{C}$ and then rinsing the material with PBS before implantation. Enhancement of the CorMatrix-ECM material with bFGF was evaluated before implantation by Western blot as previously described. ${ }^{4}$

\section{Epicardial Infarct Repair Procedure}

Successfully infarcted animals were randomly assigned to 1 of 3 groups: nonenhanced epicardial infarct repair, bFGF-enhanced epicardial infarct repair, or sham procedure. Three weeks post-MI, rats were anesthetized and maintained as described above. The heart was exposed via a left anterolateral thoracotomy 1 intercostal space caudal to the previous incision. The infarcted region was identified visually. Animals randomized to 1 of the treatment groups received either nonenhanced or bFGF-enhanced CorMatrix-ECM patch sewn onto the epicardial surface of the infarct using a continuous 7-0 polypropylene suture. Animals randomized to the sham group underwent a similar suture technique performed without securing a CorMatrix-ECM patch. The incision was reapproximated and the animals were recovered. Analgesia, antibiotic prophylaxis, and preoperative fluids were administered as described above.

\section{Histology and Immunohistochemistry}

Animals were euthanized and hearts explanted 16 weeks after treatment. The left ventricle was isolated and long axis sections were fixed in $10 \%$ neutral buffered formalin and embedded in paraffin. Samples were stained with hematoxylin and eosin or Masson's trichrome to evaluate integration of the CorMatrix-ECM patch material. Autofluorescent background signals was reduced using $50 \mathrm{mM}$ ammonia in $70 \%$ ethanol for 180 minutes, followed by 5 minutes in $0.1 \%$ Sudan black B in $70 \%$ ethanol. ${ }^{5}$ Antigen retrieval was performed on all samples before immunofluorescent staining by incubating slides in $10 \mathrm{mM}$ citric acid in $0.05 \%$ Tween detergent at a pH of 6.0 for 60 minutes at $95^{\circ} \mathrm{C}$ to $100^{\circ} \mathrm{C}$. Proteinase
$\mathrm{K}$ antigen retrieval protocol was used on collagen type I antibodies, in addition to citric acid antigen retrieval. TE buffer $(50 \mathrm{mM}$ Tris base, $1 \mathrm{mM}$ thylenediaminetetraacetic acid, $0.5 \%$ Triton $\mathrm{X}-100, \mathrm{pH} 8.0$ ) with proteinase $\mathrm{K}$ $(20 \mu \mathrm{g} / \mathrm{mL})$ was incubated for 20 minutes at $37^{\circ} \mathrm{C}$ in a humidified chamber and washed off with TE buffer in 5\% goat serum. Samples were blocked with $5 \%$ goat serum $0.1 \%$ Triton X-100 in PBS for 1 hour, then stained using primary troponin (Developmental Studies Hybridoma Bank, Iowa City, Iowa), collagen type I (Abcam, Cambridge, Mass), alpha smooth muscle actin (Sigma-Aldrich, St Louis, Mo), and basic-fibroblast growth factor antibodies (Santa Cruz Biotechnology, Inc, Dallas, Tex). Nuclei were stained with 4'6-diamino2-phenylindol. Stained specimens were examined using a Zeiss Axiovert 40X light microscope (Carl Zeiss Canada, Ontario, Canada) and a Zeiss LSM-5 Laser Confocal Microscope (Carl Zeiss GmBH, Heidelberg, Germany).

\section{Echocardiography}

Echocardiographic evaluation was performed 2 weeks post-infarct (as a baseline before treatment), and between 16 and 19 weeks after treatment. All echocardiograms were performed under isoflurane inhalational anesthesia in the dorsal decubitus position, and recorded using an Esaote MyLab30 Gold Cardiovascular Ultrasound system (Canadian Veterinary Imaging, Georgetown, Ontario, Canada). Animals with an ejection fraction $>60 \%$ measured by echocardiography were not randomized.

\section{Pressure Volume Loop Analysis}

Hemodynamic parameters were further assessed using a pressure volume loop system 16 to 19 weeks after treatment. A $2 \mathrm{~F}$ conductance catheter (SPR-838 or SPR849, Millar Instruments, Houston, Tex) was inserted into the left ventricle via the right carotid artery. The appropriate catheter length was chosen based on the long axis measurement of the left ventricle determined via echocardiography. Left ventricular pressure and volume data was collected and analyzed as previously described ${ }^{6}$ using ADInstruments software (Colorado Springs, Colo).

\section{Ex Vivo Passive Left Ventricle Pressure-Volume Assessment}

After diastolic arrest, a latex balloon coupled to a pressure catheter was inserted into the left ventricle and passive pressure distention measures were performed as previously described. ${ }^{7}$ Left ventricle volume was determined by the volume of saline injected into the balloon to achieve $30 \mathrm{~mm} \mathrm{Hg}$

\section{Statistical Analysis}

All data are expressed as mean \pm standard deviation. Prism 5.0d (GraphPad Software Inc, La Jolla, Calif) statistical software was used for all statistical analysis. Data was analyzed by 1-way analysis of variance, repeated measures analysis of variance, and unpaired $t$ test where appropriate. Multiple comparisons were performed using the Bonferroni method.

\section{RESULTS}

A total of 56 rats were evaluated in this study (normal, $\mathrm{n}=13$; nonenhanced ECM, $\mathrm{n}=18$; bFGF-enhanced ECM, $\mathrm{n}=10$; and sham, $\mathrm{n}=15$ ). No animals were lost during the period of follow-up. All animals had serial echocardiography assessments. A random sample of 5 per group received histologic analysis. A random sample of animals received invasive hemodynamic assessments and passive pressure-volume analysis without histologic analysis (normal, $\mathrm{n}=8$; nonenhanced $\mathrm{ECM}, \mathrm{n}=7$; bFGF-enhanced ECM, $\mathrm{n}=5$; and sham, $\mathrm{n}=6$ ). 

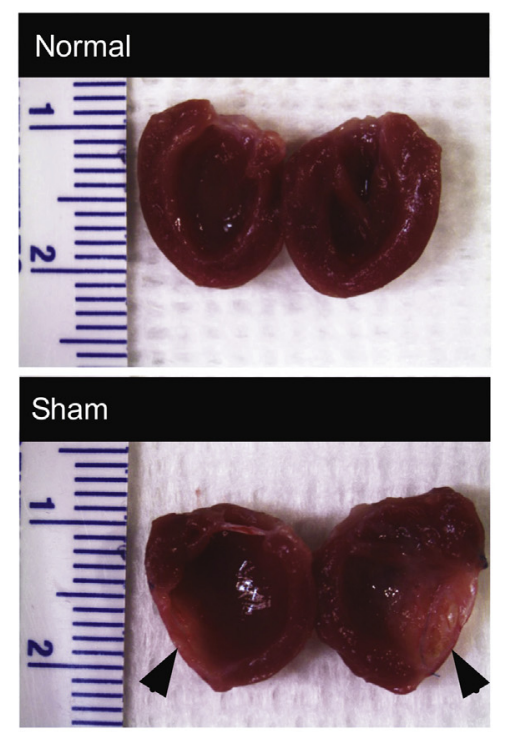

Non-Enhanced
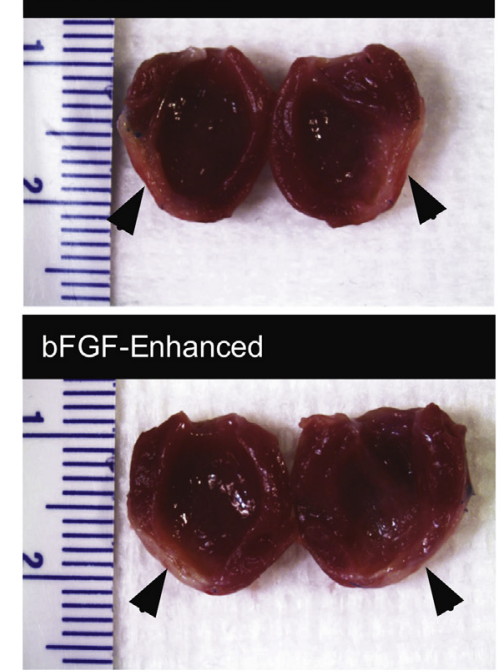
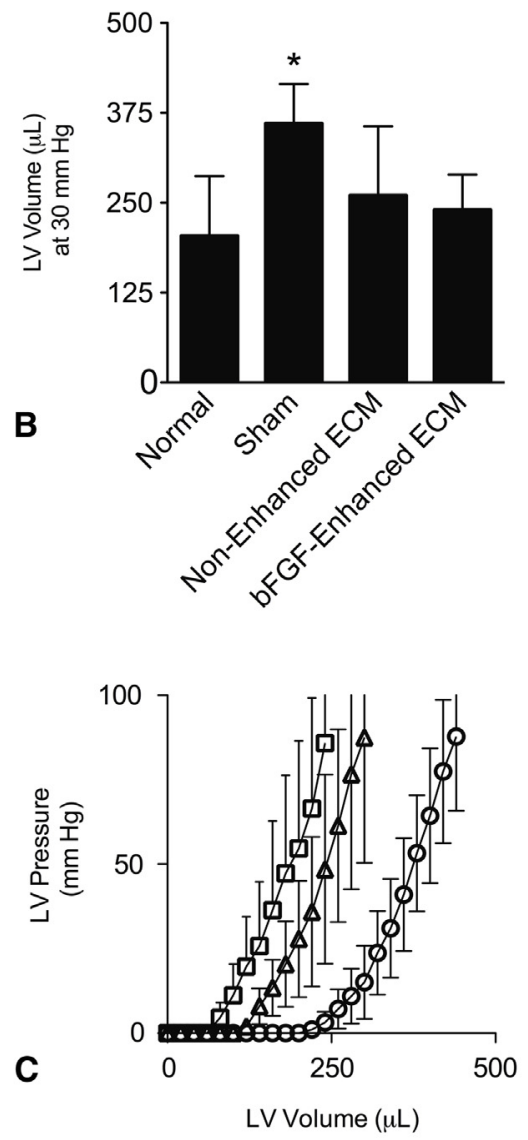

\begin{tabular}{ll}
\hline$\square$ & Normal \\
$\Delta-$ & Non-Enhanced \\
○- & Sham
\end{tabular}

FIGURE 1. A, Left ventricles $(L V s)$ transected longitudinally through the anterior and posterior walls to reveal the infarcted myocardium within the anterior wall (indicated by the arrowheads). B, Passive pressure distension was performed in explanted LVs from normal, sham, nonenhanced CorMatrix-ECM patch (CorMatrix Cardiovascular Inc, Roswell, Ga), and basic fibroblast growth factor ( $b F G F$ )-enhanced CorMatrix-ECM patch animals ( $\mathrm{n}=5$ per group). The $\mathrm{LV}$ volume at $30 \mathrm{~mm} \mathrm{Hg}$ was recorded and compared between groups as an index of chamber size. C, Passive pressure-volume curves from normal, nonenhanced, and sham animals are shown and confirm that chamber compliance was similar between groups. $* P<.05$. ECM, Extracellular matrix.

Gross examination of explanted hearts revealed more extensive regional scarring in sham animals compared with non-enhanced and bFGF-enhanced CorMatrix-ECMtreated animals (Figure 1, A). The infarct region was thinner in sham animals compared with both nonenhanced and bFGF-enhanced CorMatrix-ECM patch animals, with the appearance of healthy myocardium toward the endocardial surface under the patch in bFGF-enhanced CorMatrix-ECM patch animals. By gross examination the left vetricle appeared dilated in sham animals compared with normal, nonenhanced and bFGF-enhanced CorMatrix-ECM patch animals. This observation was confirmed by ex vivo passive pressure measures of left ventricle volumes, which demonstrated an increase in left ventricle volumes for sham animals compared with normal, nonenhanced, and bFGFenhanced CorMatrix-ECM-treated animals $(360.0 \pm 54.8$ $\mu \mathrm{L}$ vs $204.0 \pm 83.0 \mu \mathrm{L}, 260.0 \pm 95.9 \mu \mathrm{L}$, and $240.0 \pm$ $49.0 \mu \mathrm{L} ; P=.02$ ) (Figure $1, B$ ). Histologic examination of explanted LV myocardium demonstrated integration of the CorMatrix-ECM material with host myocardium (Figure 2, A). No evidence of an immune reaction to the material was observed 16 weeks post-treatment. Integration was also assessed by immunohistochemistry (Figure 2, B), which showed the ECM transition from the host to the 

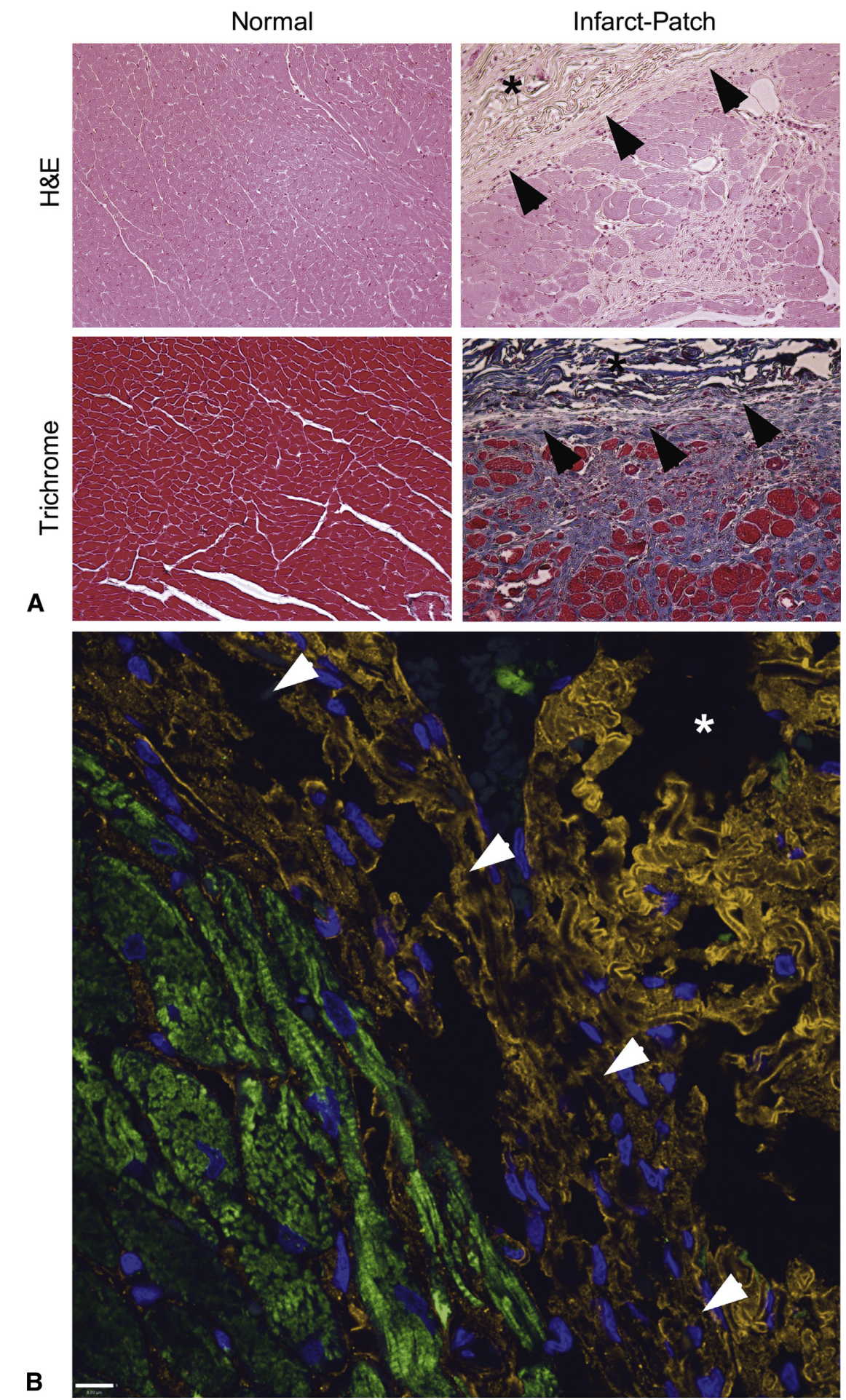

FIGURE 2. Myocardial tissue from a nonenhanced CorMatrix-ECM patch (CorMatrix Cardiovascular Inc, Roswell, Ga) animal. A, Histology. B, Immunohistochemistry. Arrowheads outline the myocardium-patch interface, green color indicates troponin, orange color indicates collagen, and blue indicates nucleus. *CorMatrix-ECM patch material.

implanted patch was free of scarring or inflammatory reactivity. Healthy troponin expressing myocytes were observed within the infarct area below the CorMatrix-ECM patch suggesting muscle preservation.
Immunohistochemistry for Type-I collagen highlighted the architecture of ECM in healthy myocardium (Figure 3, A) compared with infarcted myocardium in sham animals (Figure 3,B), non-enhanced CorMatrix-ECM 

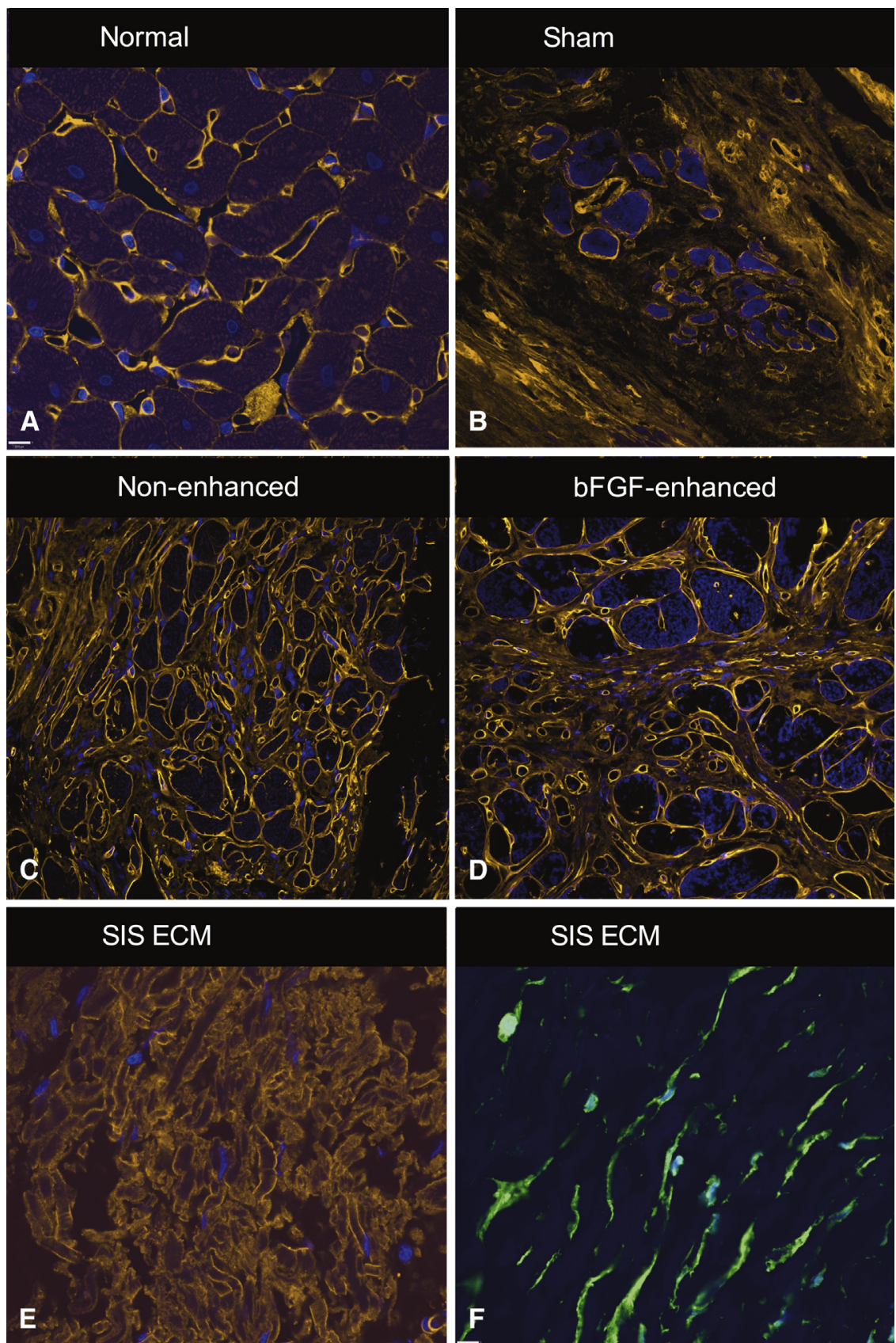

FIGURE 3. Immunohistochemistry. A, Normal myocardium. B, Infarcted myocardium from a sham. C, Nonenhanced CorMatrix-ECM patch (CorMatrix Cardiovascular Inc, Roswell, Ga). D, Basic fibroblast growth factor ( $b F G F)$-enhanced CorMatrix-ECM patch animal stained with anti-collagen (orange) and 4'6-diamino2-phenylindol (blue). E, In vivo CorMatrix-ECM patch stained with anti-collagen (orange). F, In vivo CorMatrix-ECM patch stained with anti-alpha-smooth muscle actin (green) and 4'6-diamino2-phenylindol (blue). SIS ECM, Small intestine submucosa extracellular matrix.

(Figure 3,C) and bFGF-enhanced CorMatrix-ECM-treated animals (Figure 3,D). Collagen matrix within the infarct area of sham animals appeared denser than CorMatrixECM-treated animals, suggesting ECM dysregulation. The cardiac ECM architecture appeared more preserved in both the nonenhanced and bFGF-enhanced CorMatrixECM-treated animals, also supporting improving ECM homeostasis. In addition, the acellular implant was repopulated with host cells suggesting de novo tissue formation in the infarct region. Immunohistochemistry of the CorMatrix-ECM patch in vivo demonstrated incorporation of host cells as represented by frequent nuclei (blue) present throughout the CorMatrix-ECM implant material (Figure 3, $E$ ). The cells within the implanted material stained positive for $\alpha$-smooth muscle actin, possibly reflecting vascular smooth muscle cells, (myo) fibroblasts or others (Figure 3,F). 

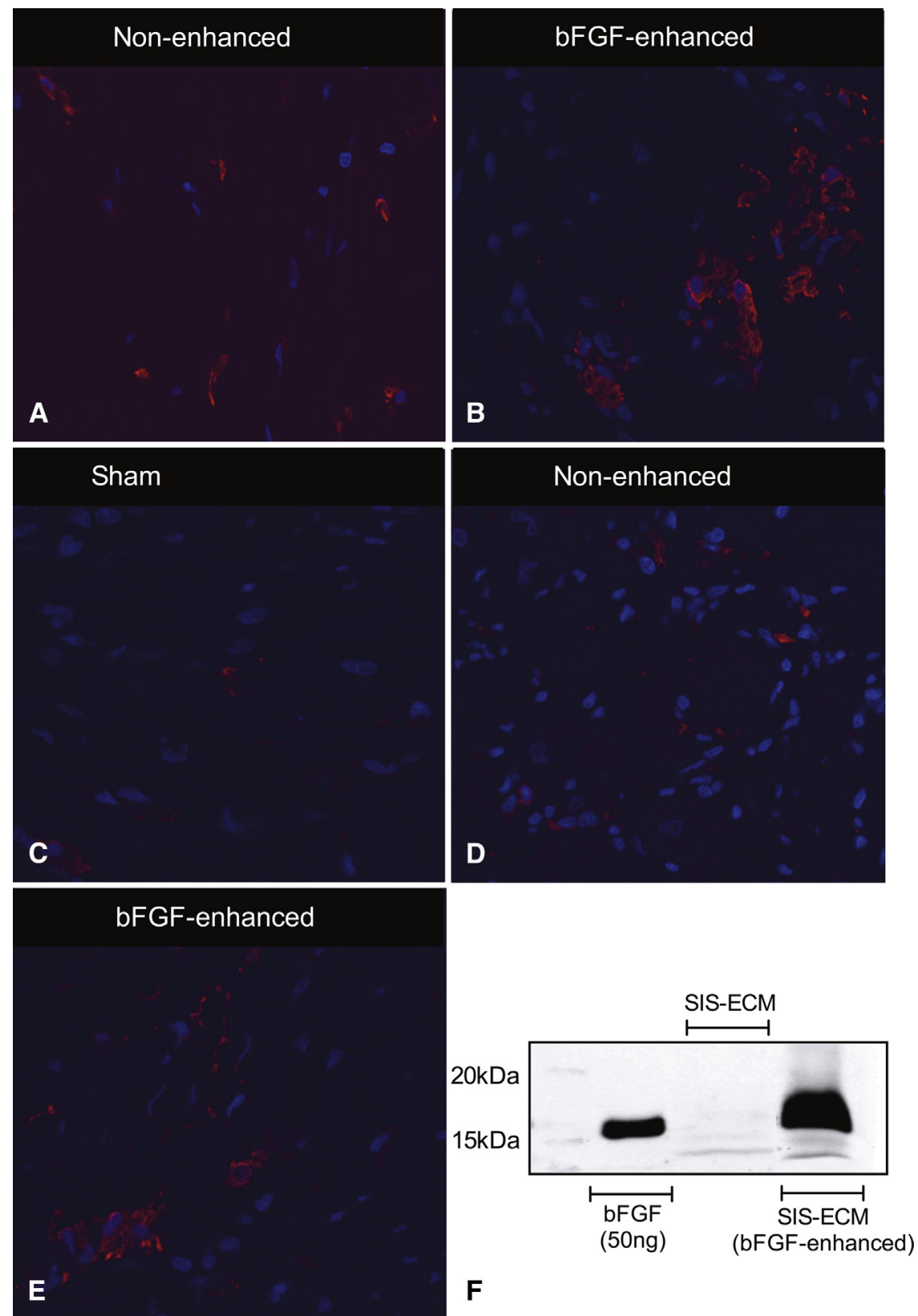

$\mathbf{F}$

FIGURE 4. CorMatrix-ECM patch (CorMatrix Cardiovascular Inc, Roswell, Ga) animal stained with anti-basic fibroblast growth factor (bFGF) (red) and 4'6-diamino2-phenylindol (blue). A, In vivo nonenhanced. B, bFGF-enhanced. C, CorMatrix-ECM and infarcted myocardium from a sham. D, Nonenhanced. E, bFGF-enhanced. F, Western blot for bFGF performed on the bFGF-enhanced CorMatrix-ECM patch before implantation. SIS ECM, Small intestine submucosa extracellular matrix.

Immunohistochemistry for bFGF expression 16 weeks after epicardial implantation demonstrated increased bFGF expressing cells within the bFGF-enhanced CorMatrixECM compared with nonenhanced CorMatrix-ECM (Figure 4, $A$ and $B$ ). Evaluation of bFGF concentration following the in vitro enhancement procedure demonstrated a marked uptake of bFGF within the CorMatrix-ECM biomaterial before epicardial implantation (Figure 4,F). Notably, after 16 weeks in vivo, bFGF expression within the CorMatrix-ECM implant localized to cell membranes suggesting that late bFGF expression was the result of bFGF-expressing host cells that infiltrated the CorMatrixECM material rather than nonspecific residual bFGF from the earlier enhancement procedure. bFGF was also upregulated within the infarcted myocardium of nonenhanced CorMatrix-ECM and to an even greater extent in bFGFenhanced CorMatrix-ECM-treated animals when compared with shams (Figure 4,C-E). These data support the 


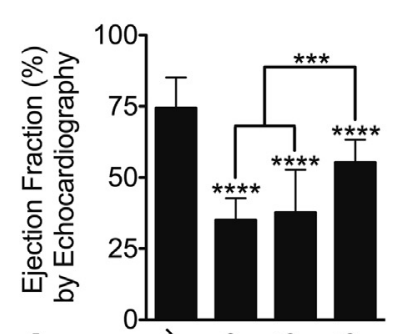

A
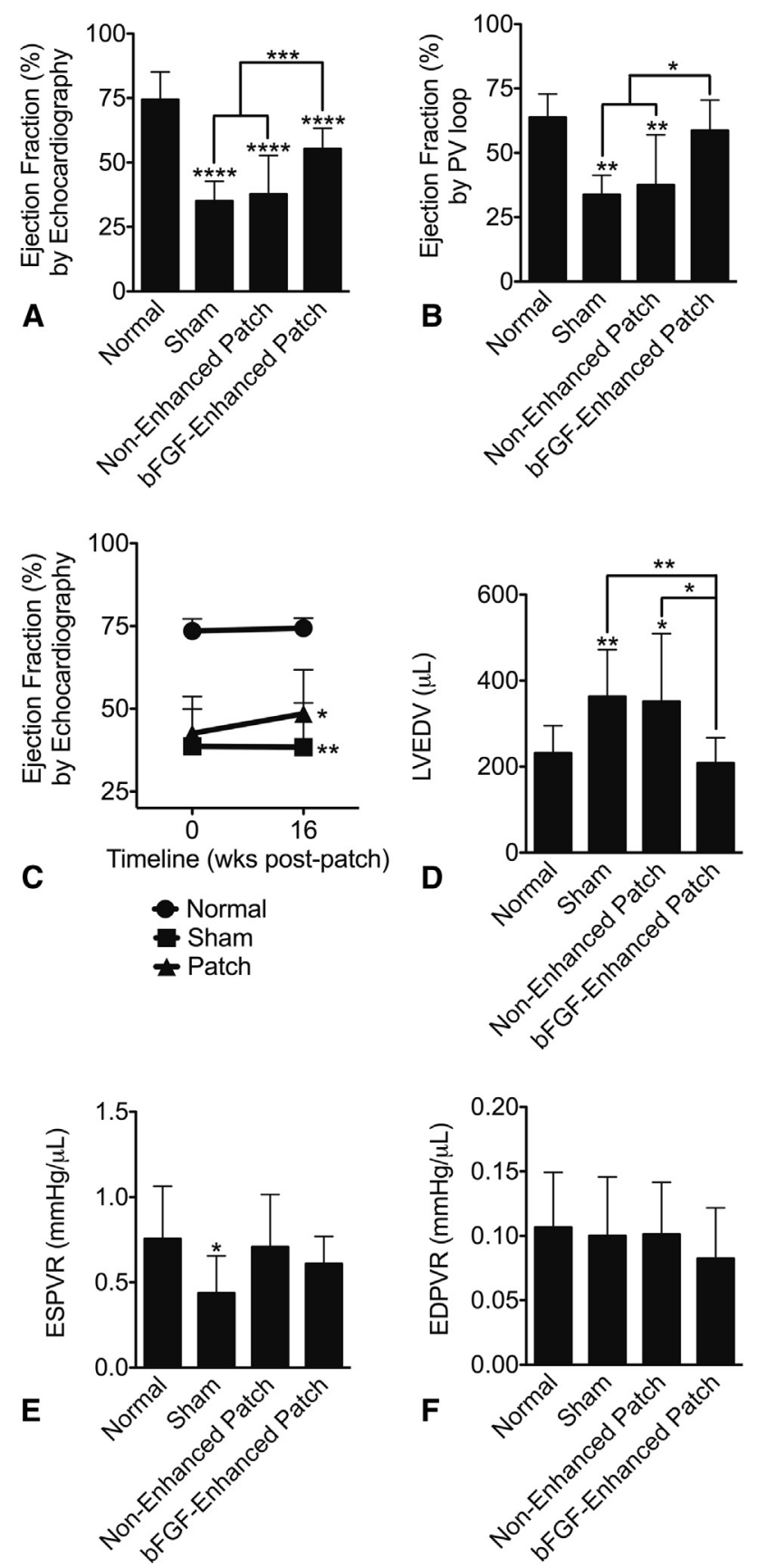

FIGURE 5. Hemodynamic parameters. A, Ejection fraction measured 16 weeks post-treatment by echocardiography in normal $(\mathrm{n}=13)$, sham $(\mathrm{n}=15)$, nonenhanced CorMatrix-ECM patch (CorMatrix Cardiovascular Inc, Roswell, $\mathrm{Ga})(\mathrm{n}=18)$, and basic fibroblast growth factor $(b F G F)$ enhanced CorMatrix-ECM patch $(\mathrm{n}=10)$ animals. B, Pressure-volume $(P V)$ loop analysis in normal $(\mathrm{n}=8)$, sham $(\mathrm{n}=6)$, nonenhanced CorMatrix-ECM patch $(\mathrm{n}=7)$, and bFGF-enhanced CorMatrix-ECM patch $(n=5)$ animals. C, Changes in ejection fraction measured by echocardiography over the time course of the experiment in normal $(n=13)$, sham $(n=15)$, and CorMatrix-ECM patch $(n=28$; patch group includes non-enhanced and bFGF-enhanced CorMatrix-ECM patch) animals. D, Left ventricular end diastolic volume ( $L V E D V$ ) measured by PV loop 16 weeks post-treatment. E, Contractility was reflected by the end systolic concept of matricellular protein interactions after regional implantation.

Echocardiography demonstrated an improvement in ejection fraction over 16 weeks in bFGF-enhanced CorMatrix-ECM-treated animals compared with shams $(55.3 \% \pm$ $8.0 \%$ vs $35.1 \% \pm 7.6 \% ; P<.001$ ) (Figure $5, A$ ). The improvement in ejection fraction was also confirmed by pressure volume loop analysis $(58.7 \% \pm 11.2 \%$ vs $33.8 \% \pm 7.5 \% ; P<.001$ ) (Figure 5, B). A consistent improvement in ejection fraction measured by serial echocardiography was observed in CorMatrix-ECM-treated animals over the 16-week observation period (Figure 5, C). Left ventricular end diastolic volumes approached normal hearts in bFGF-enhanced CorMatrix-ECM animals and but were significantly increased in both nonenhanced CorMatrix-ECM-treated and sham animals $(208.0 \pm 59.3 \mu \mathrm{L}$ vs $351.5 \pm 157.7 \mu \mathrm{L}$ and $363.1 \pm 108.7 \mu \mathrm{L} ; P<.01)$ (Figure $5, D$ ). These data indicate left ventricle dilation in sham and nonenhanced CorMatrix-ECM-treated animals that was prevented in bFGF-enhanced CorMatrix-ECMtreated animals. Cardiac output and stroke volume was equivalent between normal, sham, nonenhanced, and bFGF-enhanced CorMatrix-ECM-treated animals (cardiac output: $46.2 \pm 16.7 \mathrm{~mL} /$ minute, $38.5 \pm 13.8 \mathrm{~mL} /$ minute, $35.2 \pm 10.5 \mathrm{~mL} /$ minute, and $37.8 \pm 10.4 \mathrm{~mL} /$ minute, respectively; $P=.442$; and stroke volume: $0.15 \pm 0.05$ $\mathrm{mL}, 0.12 \pm 0.05 \mathrm{~mL}, 0.11 \pm 0.03 \mathrm{~mL}$, and $0.12 \pm 0.03$ $\mathrm{mL}$, respectively; $P=.39$ ) indicating that none of the animals, despite a substantial transmural infarct, developed overt heart failure at 16 weeks.

The end-systolic pressure volume relationship, an index of myocardial contractility, demonstrated preservation of systolic function in both non-enhanced and bFGFenhanced CorMatrix-ECM-treated animals compared with shams $(0.709 \pm 0.306$ and $0.609 \pm 0.160$ vs $0.437 \pm$ $0.218 ; P<.05$ ) (Figure 5, E). The infarct area appeared smaller and wall thickness increased in both nonenhanced CorMatrix-ECM-treated and bFGF-enhanced CorMatrixECM-treated animals compared with shams (Figure 1, A), suggesting that improved contractility after epicardial infarct repair may be the result of decreased infarct expansion.

Left ventricular compliance was evaluated by left ventricular end diastolic pressure measures, the end-diastolic pressure volume relationship, and by passive pressurevolume curves. Left ventricular end diastolic pressure was equivalent in CorMatrix-ECM-treated (nonenhanced and bFGF-enhanced) and sham animals $(10.2 \pm 3.2 \mathrm{~mm} \mathrm{Hg}$

pressure volume relationship (ESPVR) measured by PV loop 16 weeks post-treatment. F, Ventricular compliance was reflected by the end diastolic pressure volume relationship (EDPVR) measured by PV loop 16 weeks post-treatment. $* P<.05$. $* * P<.01$. $* * * P<.001$. $* * * * P<.0001$. 
and $11.7 \pm 1.4 \mathrm{~mm} \mathrm{Hg}$ vs $12.9 \pm 3.1 \mathrm{~mm} \mathrm{Hg} ; P=.45)$. In addition, the end-diastolic pressure volume relationship was also equivalent across study groups $(0.10 \pm 0.04$ and $0.08 \pm$ 0.04 vs $0.10 \pm 0.5 ; P=.79$ ) (Figure $5, F$ ), indicating that epicardial infarct repair does not alter left ventricle compliance or restrict left ventricle filling. Passive pressurevolume curves (Figure 1,C) showed similar chamber compliance between untreated ECM and normal hearts also indicating that epicardial CorMatrix-ECM therapy does not restrict ventricular expansion.

\section{DISCUSSION}

ECM influences post-MI structural remodeling by providing structural support to tissues. ECM also provides a dynamic microenvironment that regulates cardiac cell morphology, differentiation, migration, and proliferation. ${ }^{8}$ ECM homeostasis is maintained by resident cardiac fibroblasts. ${ }^{9}$ Ischemic insults trigger conversion of quiescent fibroblasts to an active myofibroblast phenotype. ${ }^{10}$ Myofibroblasts alter ECM homeostasis by increasing ECM turnover and stimulating scar formation to reinforce the damaged region and prevent mechanical complications such as myocardial rupture. ${ }^{9}$ Remodeling of ECM early after MI is adaptive, but persistent ECM dysregulation results in progressive myocardial fibrosis, ventricular dilation, and heart failure. ${ }^{11,12}$ Matricellular proteins ${ }^{13}$ within the ECM play a key role in regulating the microenvironment of cardiac fibroblasts ${ }^{7}$ and in so doing, serve as a potential therapeutic target for novel interventions aimed at mitigating fibrosis. We believe that targeting ECM dysregulation and restoring key matricellular proteins after MI may improve myocardial repair and prevent maladaptive structural remodeling.

Tissue engineering approaches aiming to mimic and replace native tissue have utilized synthetic or decellularized xenogenic ECM scaffolds with targeted ex vivo cell repopulation. $^{11,14-19}$ Acellular biologic scaffolds are preferable over synthetic material as they promote host cellular repopulation, tissue regeneration, growth, and adaptive remodeling after implantation. ${ }^{20,21}$ We evaluated a novel surgical approach that leverages a unique ECM biomaterial (ie, CorMatrix-ECM) in combination with a paracrine factor (ie, bFGF) capable of preventing maladaptive fibrosis and mobilizing endogenous regenerative cell populations. We believe CorMatrix-ECM is an ideal platform technology for epicardial infarct repair given its biocompatibility, well-established safety profile, and immediate commercial availability for use in cardiac surgery. CorMatrix-ECM is a biologic porcine matrix scaffold that retains its native 3-dimensional collagen architecture and key matricellular proteins, including glycosaminoglycans, bFGF, fibronectin, and laminin. ${ }^{22-25}$ The potential of this biomaterial has been clinically validated in several noncardiac applications. ${ }^{20,21}$ Although the biomaterial is xenogenic, in vivo studies have confirmed excellent host immune tolerance. ${ }^{26,27}$ To date, it has been used safely and effectively in numerous routine cardiac applications. ${ }^{28}$

In our study we demonstrate beneficial effects of CorMatrix-ECM biomaterial patch on post-MI cardiac remodeling. Epicardial application of CorMatrix-ECM preserves native ECM architecture within an infarcted myocardium and decreases regional scar formation. Epicardial implantation of CorMatrix-ECM alters ECM homeostasis toward reduced fibroblast activation and fibrosis as suggested by the observed increase in bFGF expression within the CorMatrix-ECM patch and host myocardium. We demonstrate that enhancing CorMatrixECM with bFGF before implantation can further improve ECM homeostasis after infarction resulting in improved chamber remodeling and cardiac function. Prevention of left ventricle dilation likely resulted in the improved ejection fraction observed with bFGF-enhanced epicardial infarct repair. Regional infract scar was reduced in CorMatrix-ECM-treated animals suggesting that epicardial infarct repair prevents infarct expansion and preserves contractile muscle. This proposed mechanism is supported by the improved myocardial contractility observed in CorMatrix-ECM-treated animals.

Our results illustrate a proof-of-concept for this novel surgical procedure. Further validation of this novel therapy in a preclinical large animal model is required. Clinical safety concerns cannot be addressed in a translational rodent study. However, we show that left ventricle compliance was not altered by the procedure, thereby mitigating potential concerns about ventricular restriction from the epicardial device implantation. These data are consistent with the histologic analysis that shows an absence of inflammation and scarring around the implant. Importantly, these observations also confirm that the reduced chamber volumes were not secondary to deleterious myocardial restriction preventing physiologic chamber expansion.

Current surgical approaches for treatment of the ischemic remodeled heart include surgical ventricular restoration or epicardial-based ventricular restraint devices, designed to reduce $L V$ volumes and subsequently improve ejection fraction. The clinical benefits of these strategies remain controversial. $^{29,30}$ Epicardial infarct repair offers a surgical alternative that, unlike surgical ventricular restoration or ventricular restraint devices, utilizes a biologically active material capable of limiting the ischemic remodeling process.

In this emerging era of tissue engineering and biosurgical approaches to heart failure, we believe that passive girdling with inert/synthetic patch materials should not be the primary goal of new therapies, recognizing that regional inert epicardial infarct patches have shown short-term benefits in animal models. To date, we have not seen effective clinical translation of therapies using synthetic and inert materials for epicardial restraint despite substantial efforts. Passive 
epicardial restraint can be detrimental and an induction of epicardial adhesions and scarring should be avoided. We believe that synthetic inert patches have these complications and would not be capable of the responses observed with CorMatrix ECM. Given that we noted a superior response with bFGF enhancement compared with untreated patches alone, these data suggest that the mechanism of benefit with the enhanced biomaterial is due to active molecular and cellular responses as opposed to purely a passive effect from adhesions and scarring given that both groups had identical patches. Future studies could be performed to better characterize the underlying mechanisms of benefit in the attenuation of remodeling observed after epicardial infarct repair with CorMatrix-ECM biomaterial patch. We show that epicardial infarct repair restores ECM homeostasis, preventing the underlying pathology that leads to ventricular dilation following MI. We believe this platform technology is clinically feasible and specifically targets the active cellular and molecular changes that underlie ischemic cardiomyopathy. Given our complete set of data, there is no suggestion of chamber restriction due to scarring or adhesion formation. This is an important distinction to passive restraint due to chamber restriction from scarring. Our data is consistent with the idea that an active process of maladaptive chamber remodeling was attenuated over time resulting in reduced chamber size. In a clinical setting, epicardial infarct repair could be feasibly introduced as an adjunct to surgical revascularization for patients with regional ischemic myocardial injury at risk of progressive maladaptive remodeling. In a recent multicenter trial, 5\% of patients admitted with an acute coronary syndrome received coronary artery bypass grafting surgery during the index hospitalization and over $10 \%$ of patients with acute coronary syndrome had coronary bypass graft surgery within 12 months of the event. ${ }^{31}$ More than 1.4 million patients are admitted to hospitals with acute coronary syndrome each year in the United States, supporting the idea that a significant subset of patients has surgical revascularization within the timeline employed by the design of our study. These patients are at higher risk for incomplete revascularization and the subacute infarct region is ignored at the time of coronary artery bypass graft. We believe this is an opportunity to treat the progressive and dynamic infarct region. Based on the functional and structural improvements observed in this study, we believe that epicardial infarct repair as an adjunct to coronary bypass grafting may be capable of reversing ischemic remodeling, improving long-term clinical outcomes compared with coronary artery bypass or percutaneous coronary intervention alone. The ideal candidates are patients with viable myocardial regions early after injury that faces the risk of progressive post-MI remodeling despite revascularization. Further preclinical studies will help optimize the potential clinical scenarios and patient populations to target with this innovation.
Previous studies aimed at developing functional myocardial constructs for use after ischemic injury have utilized ex vivo scaffold-based stem cell repopulation techniques. ${ }^{15,19,32}$ The clinical applicability of this approach is limited by the sparse availability of autologous stem cells, possible immunosuppressive medication protocols, and the lack of an "off-the-shelf" approach. Implantation of a commercially available biocompatible acellular scaffold capable of being repopulated with endogenous cells in vivo would eliminate these barriers to adoption. We demonstrate that host cells are capable of a robust repopulation of the acellular CorMatrix-ECM biomaterial when implanted onto the epicardial surface of a post-MI heart. Previous studies demonstrate that enhancement of biologic ECM scaffolds with matricellular proteins increase the efficiency of scaffold-based cellular repopulation. ${ }^{33,34}$ Our data support this conclusion, demonstrating an increase in bFGFexpressing cells within the CorMatrix-ECM biomaterial when enhanced with bFGF before implantation. These data highlight the ability to achieve in vivo tissue regeneration without the need for exogenous cell repopulation.

Although bFGF is known to decrease fibrosis, its clinical administration has remained problematic. Epicardial infarct repair offers a feasible approach for regional tissue-specific delivery of bFGF as an antifibrotic myocardial treatment strategy. We believe epicardial infarct repair is a promising platform technology that can be tailored to the individual patient. We aim to explore material enhancement with additional matricellular proteins known to regulate tissue remodeling after injury, such as endogenous tissue inhibitors of metalloproteinases to suppress ECM turnover ${ }^{35}$ and vascular endothelial growth factor, to stimulate angiogenesis. ${ }^{36,37}$

There are a number of study limitations to consider. First, animals were matched for infarct size using functional data that were not confirmed by histologic analysis. Second, wall thickness was not quantitatively assessed and compared between groups, only a qualitative assessment was provided. Third, the rodent infarct model may not be reflective of the human condition. These issues may be best addressed with further validation in large preclinical animal models.

\section{CONCLUSIONS}

Epicardial infarct repair favorably alters cardiac remodeling post-MI, mitigating ischemic heart failure. These data establish proof-of-concept for this novel surgical procedure in a small animal model. Epicardial infarct repair should be further validated in a preclinical model before translation into human patients.

\section{References}

1. Go AS, Mozaffarian D, Roger VL, Benjamin EJ, Berry JD, Borden WB, et al. Executive summary: heart disease and stroke statistics—2013 update: a report from the American Heart Association. Circulation. 2013;127:143-52. 
2. Canadian Council on Animal Care. Guide for the care and use of experimental animals. Available at: http://www.ccac.ca/Documents/Standards/Guidelines/ Experimental_Animals_Vol1.pdf. Accessed August 30, 2013.

3. National Academy of Sciences. Guide for the Care and Use of Laboratory Animals. Washington, DC: The National Academy Press; 1996.

4. Fedak PWM, Bai L, Turnbull J, Ngu J, Narine K, Duff HJ. Cell therapy limits myofibroblast differentiation and structural cardiac remodeling: basic fibroblast growth factor-mediated paracrine mechanism. Circ Heart Fail. 2012;5:349-56.

5. Oliveira VC, Carrara RCV, Simoes DLC, Saggioro FP, Carlotti CG, Covas DT, et al. Sudan Black B treatment reduces autofluorescence and improves resolution of in situ hybridization specific fluorescent signals of brain sections. Histol Histopathol. 2010;25:1017-24.

6. Pacher P, Nagayama T, Mukhopadhyay P, Bátkai S, Kass DA. Measurement of cardiac function using pressure-volume conductance catheter technique in mice and rats. Nat Protoc. 2008;3:1422-34.

7. Ohno N, Fedak PWM, Weisel RD, Mickle DAG, Fujii T, Li R- K. Transplantation of cryopreserved muscle cells in dilated cardiomyopathy: effects on left ventricular geometry and function. J Thorac Cardiovasc Surg. 2003;126:1537-48.

8. Murphy-Ullrich JE. The de-adhesive activity of matricellular proteins: is intermediate cell adhesion an adaptive state? J Clin Invest. 2001;107:785-90.

9. Brown RD, Ambler SK, Mitchell MD. The cardiac fibroblast: therapeutic target in myocardial remodeling and failure. Annu Rev Pharmacol Toxicol. 2005;45: 657-87.

10. Rosenkranz S. TGF-beta1 and angiotensin networking in cardiac remodeling. Cardiovasc Res. 2004;63:423-32.

11. Graham HK, Horn M, Trafford AW. Extracellular matrix profiles in the progression to heart failure. Acta Physiologica. 2008;194:3-21.

12. Opie LH, Commerford PJ, Gersh BJ, Pfeffer MA. Controversies in ventricular remodeling. Lancet. 2006;367:356-67.

13. Frangogiannis NG. Matricellular proteins in cardiac adaptation and disease. Physiol Rev. 2012;92:635-88.

14. Khouw IM, van Wachem PB, Plantinga JA, Vujaskovic Z, Wissink MJ, de Leij LF, et al. TGF-beta and bFGF affect the differentiation of proliferating porcine fibroblasts into myofibroblasts in vitro. Biomaterials. 1999;20:1815-22.

15. Ott HC, Matthiesen TS, Goh S-K, Black LD, Kren SM, Netoff TI, et al. Perfusion-decellularized matrix: using nature's platform to engineer a bioartificial heart. Nat Med. 2008;14:213-21.

16. Fedak PWM. Cardiac progenitor cell sheet regenerates myocardium and renews hope for translation. Cardiovasc Res. 2010;87:8-9.

17. Fedak PWM, Szmitko PE, Weisel RD, Altamentova SM, Nili N, Ohno N, et al. Cell transplantation preserves matrix homeostasis: a novel paracrine mechanism. J Thorac Cardiovasc Surg. 2005;130:1430-9.

18. Fazel S, Chen L, Weisel RD, Angoulvant D, Seneviratne C, Fazel A, et al. Cell transplantation preserves cardiac function after infarction by infarct stabilization: augmentation by stem cell factor. J Thorac Cardiovasc Surg. 2005;130; 1310 .

19. Badylak S, Freytes D, Gilbert T. Extracellular matrix as a biological scaffold material: structure and function. Acta Biomaterialia. 2009;5:1-13.

20. Robinson KA, Li J, Mathison M, Redkar A, Cui J, Chronos NAF, et al. Extracellular matrix scaffold for cardiac repair. Circulation. 2005;112:I135-43.
21. Zhang Y, Frimberger D, Cheng EY, Lin H-K, Kropp BP. Challenges in a larger bladder replacement with cell-seeded and unseeded small intestinal submucosa grafts in a subtotal cystectomy model. BJU Int. 2006;98:1100-5.

22. Brown B, Lindberg K, Reing J, Stolz DB, Badylak SF. The basement membrane component of biologic scaffolds derived from extracellular matrix. Tissue Eng. 2006;12:519-26.

23. McDevitt CA, Wildey GM, Cutrone RM. Transforming growth factor-beta1 in a sterilized tissue derived from the pig small intestine submucosa. J Biomed Mater Res A. 2003;67:637-40.

24. Voytik-Harbin SL, Brightman AO, Kraine MR, Waisner B, Badylak SF. Identification of extractable growth factors from small intestinal submucosa. J Cell Biochem. 1997;67:478-91.

25. Hodde JP, Ernst DMJ, Hiles MC. An investigation of the long-term bioactivity of endogenous growth factor in OASIS Wound Matrix. J Wound Care. 2005;14 23-5.

26. Daly KA, Stewart-Akers AM, Hara H, Ezzelarab M, Long C, Cordero K, et al Effect of the alphaGal epitope on the response to small intestinal submucosa extracellular matrix in a nonhuman primate model. Tissue Eng Part A. 2009; 15:3877-88.

27. Mostow EN, Haraway GD, Dalsing M, Hodde JP, King D, OASIS Venus Ulcer Study Group. Effectiveness of an extracellular matrix graft (OASIS Wound Matrix) in the treatment of chronic leg ulcers: a randomized clinical trial. $J$ Vasc Surg. 2005;41:837-43.

28. Quarti A, Nardone S, Colaneri M, Santoro G, Pozzi M. Preliminary experience in the use of an extracellular matrix to repair congenital heart diseases. Interact Cardiovasc Thorac Surg. 2011;13:569-72.

29. Suma H, Anyanwu AC. Current status of surgical ventricular restoration for ischemic cardiomyopathy. Semin Thorac Cardiovasc Surg. 2012;24:294-301.

30. Costanzo MR, Ivanhoe RJ, Kao A, Anand IS, Bank A, Boehmer J, et al. Prospective evaluation of elastic restraint to lessen the effects of heart failure (PEER LESS-HF) trial. J Card Fail. 2012;18:446-58.

31. Wallentin L, Becker RC, Budaj A, Cannon CP, Emanuelsson H, Held C, et al. Ticagrelor versus clopidogrel in patients with acute coronary syndromes. $N$ Engl J Med. 2009;361:1045-57.

32. Gilbert TW, Nieponice A, Spievack AR, Holcomb J, Gilbert S, Badylak SF. Repair of the thoracic wall with an extracellular matrix scaffold in a canine model. J Surg Res. 2008;147:61-7.

33. Kang K, Sun L, Xiao Y, Li S-H, Wu J, Guo J, et al. Aged human cells rejuvenated by cytokine enhancement of biomaterials for surgical ventricular restoration. $J$ Am Coll Cardiol. 2012;60:2237-49.

34. Beattie AJ, Gilbert TW, Guyot JP, Yates AJ, Badylak SF. Chemoattraction of progenitor cells by remodeling extracellular matrix scaffolds. Tissue Eng Part A. 2009; 15:1119-25.

35. Fedak PWM, Verma S, Weisel RD, Li R-K. Cardiac remodeling and failure from molecules to man (Part II). Cardiovasc Pathol. 2005;14:49-60.

36. Fedak PWM. Paracrine effects of cell transplantation: modifying ventricular remodeling in the failing heart. Semin Thorac Cardiovasc Surg. 2008;20:87-93.

37. Kempf T, Zarbock A, Vestweber D, Wollert KC. Anti-inflammatory mechanisms and therapeutic opportunities in myocardial infarct healing. J Mol Med. 2012;90: $361-9$. 